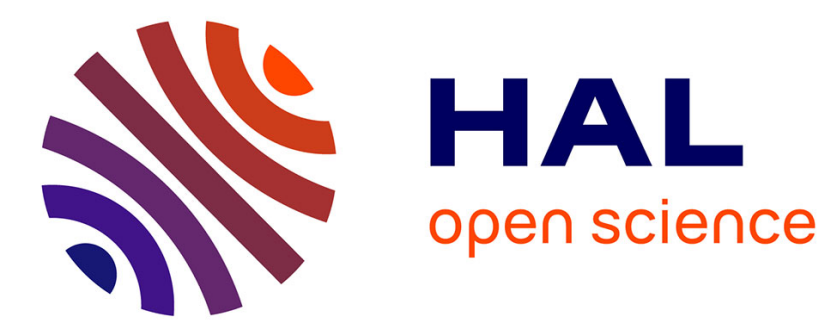

\title{
Adaptive control of a robotic exoskeleton for neurorehabilitation
}

Tommaso Proietti, Nathanael Jarrasse, Agnès Roby-Brami, Guillaume Morel

\section{To cite this version:}

Tommaso Proietti, Nathanael Jarrasse, Agnès Roby-Brami, Guillaume Morel. Adaptive control of a robotic exoskeleton for neurorehabilitation. 2015 7th International IEEE/EMBS Conference on Neural Engineering (NER), Apr 2015, Montpellier, France. pp.803-806. hal-02110265

\section{HAL Id: hal-02110265 \\ https://hal.science/hal-02110265}

Submitted on 25 Apr 2019

HAL is a multi-disciplinary open access archive for the deposit and dissemination of scientific research documents, whether they are published or not. The documents may come from teaching and research institutions in France or abroad, or from public or private research centers.
L'archive ouverte pluridisciplinaire HAL, est destinée au dépôt et à la diffusion de documents scientifiques de niveau recherche, publiés ou non, émanant des établissements d'enseignement et de recherche français ou étrangers, des laboratoires publics ou privés. 


\title{
Adaptive control of a robotic exoskeleton for neurorehabilitation
}

\author{
Tommaso Proietti, Nathanaël Jarrassé, Agnès Roby-Brami, and Guillaume Morel
}

\begin{abstract}
Neurorehabilitation efficiency increases with therapy intensity and subject's involvement during physical exercises. Robotic exoskeletons could bring both features, if they could adapt the level of assistance to patient's motor capacities. To this aim, we developed an exoskeleton controller, based on adaptive techniques, that can actively modulate the stiffness of the robotic device in function of the subject's activity. We tested this control law on one healthy subject with an upper-limb exoskeleton. The experiment consisted in learning a trajectory imposed by the robot. The early results show the different features allowed by our controller with respect to controllers commonly used for neurorehabilitation with exoskeletons.
\end{abstract}

Index Terms - Adaptive control, upper-limb robotic exoskeletons, neurorehabilitation, post-stroke robotic therapy.

\section{INTRODUCTION}

Stroke survivors are usually left with disability, mainly motor impairments on upper-limb movements and loss of hand dexterity, both partially recoverable by undergoing rehabilitation. Rehabilitation has been proven to be effective when it is both intense and involving for the patient [1]. Robotics is one possible solution to provide intensity, increasing the number of repetitions which a therapist could impose, as well as involvement thanks to technology appeal and virtual reality [2].

In the literature, the most common approach for controlling exoskeletons for neurorehabilitation involves passive control: the subject is passive while the robot forces produce the motion, eventually through impedance control with an a priori model-based compliance. However, the effectiveness of such passive motions for stimulating motor recovery is limited [3]. Active-assistive control shall thus be used, where the subject controls the motion. Here, the robot provides assistance by virtually constraining the motion and by supporting the impaired limb, based on performance indexes [4]. Ultimately, it is essential for rehabilitation robots to allow a shared control of the movements, as soon as the patient has recovered a minimal amount of motor capacity [5], [6]. Indeed, as neurorehabilitation addresses issues related to motor control, the devices must allow patients to express whatever movement they can without suppressing any motor capability [7].

In this sense, techniques from adaptive control may lead to improvements: adaptation can allow the robot to modulate its assistance level based on the subject's need. Advantageously, adaptation can also tune the controller to account of differences between patients.

The authors are with Sorbonne Universités, UPMC Univ. Paris 06, CNRS, UMR 7222, INSERM, the Institute of Intelligent Systems and Robotics (ISIR), 4 place Jussieu, 75005, Paris, France (correspondence should be addressed to jarrasse@ isir.upmc.fr)
So far few research groups have tried to introduce adaptive controllers into rehabilitation robotics with upper-limb exoskeletons. Pneu-WREX researchers developed an "assist-asneeded" model-based adaptive controller [8]. A ProportionalDerivative (PD) controller on the end-effector cooperates with an adaptive feedforward assistive term which tries to reduce simultaneously the total effort of the controller and the tracking error by the subject. Assistance-as-needed is achieved by adding a force decay term, which reduces the output from the robot when errors in task execution are small. The gains of the PD controller are fixed and chosen in order to have a soft, slightly under-damped feeling on the arm. A different approach is given by iterative learning control of RUPERT IV in [9]: here the feedforward assistive term is learnt by using trial-by-trial adaptation based on the error of the previous trial and on a complex nonlinear function involving fuzzy logic and tracking error statistics. The feedforward term is then added to a PID (PD with an Integral term) feedback controller at the joint level.

Adaptation can also exploit muscle activity measured by electromyography (EMG) to update on-line the controllers, either the feedforward term or the gains of the feedback loop/corrector [10]. This has limited application in practice, though, since EMG suffers from many practical drawbacks, such as low signal-to-noise ratio, difficulties in extracting single muscle activation profiles, influence of sweating and muscle fatigue on the output signals, etc.

The controller proposed in this paper leverages on ideas from [11] and moves them from planar robots to exoskeletons. It aims at automatically adapting from passive to active control: it stiffens up and guides the subject when he is not able to perform the task, while it relaxes when the subject has enough capacities to perform well along the trajectory. The main innovation is the continuous adaptation provided by the proposed algorithm. It allows the subject to feel assisted but never over-constrained, without manual tuning of the robot. To achieve the overall behaviour, offline trial-by-trial adaptation of control feedback gains is done.

\section{Adaptive Controller}

In human motor control, it is generally assumed that each of the motor command $\mathbf{w} \equiv\left(w_{1} \ldots w_{n}\right)^{T}$ for the $n$ muscles involved in a movement is composed of a feedforward term $\mathbf{u}$ and a feedback term $\mathbf{v}$

$$
\mathbf{w}=\mathbf{u}+\mathbf{v} .
$$

The feedforward term is learnt trial-by-trial by the central nervous system (CNS) and it has the role of performing the motion task. The feedback term helps in performing the task 
by taking into account disturbances, unexpected obstacles, contact with the environment, etc. The differences in roles explains the necessity of quickness by the CNS to correctly perform actions, since feedforward control is faster than feedback, but alone it may fail.

The proposed robot controller involves feedforward and feedback as well, where now $\mathbf{w}$ stands for the overall control torque, $\mathbf{u}$ is the feedforward torque, and feedback term $\mathbf{v}$ is provided by a PD controller

$$
\mathbf{v}=\mathbf{K}_{p} \mathbf{e}+\mathbf{K}_{d} \dot{\mathbf{e}}, \quad \mathbf{e}=\mathbf{q}_{r}(t)-\mathbf{q}(t),
$$

where $\mathbf{q}(t)$ is the joint position vector, and error signals $\mathbf{e}$ and $\dot{\mathbf{e}}$ are calculated respectively with respect to the reference joint trajectory $\mathbf{q}_{r}(t)$ and the reference joint velocity $\dot{\mathbf{q}}_{r}(t)$. $\mathbf{K}_{p}$ and $\mathbf{K}_{d}$ are positive diagonal matrices of gains.

The feedforward torque is a model-based gravity compensation, which helps the subject not to feel the weight of the robot.

The robot imposes the reference trajectory $\mathbf{q}_{r}(t)$ to the subject's joints when the stiffness $\mathbf{K}_{p}$ is high, which is suited for early rehabilitation exercises, when the subject cannot provide large forces (passive mode). In this case, the robot torques are large. After learning, the subject is expected to provide more energy in order to follow the learnt trajectory, which is assumed to lead to less torque from the robot. To achieve this adaptive behaviour, the gains of the PD controller are adapted trial-by-trial, based on the former values of the feedback term. Namely, at a given trial $k$, the controller proportional gain is computed by:

$$
\mathbf{K}_{p}^{k}=\mathbf{K}_{p}^{k-1}+\beta\left|\mathbf{v}^{k-1}\right|-\gamma
$$

where the learning parameter $\beta$ and the decay $\gamma$ are positive, and $\left|\mathbf{v}^{k-1}\right|$ represents the mean of the absolute value of feedback term during trial $k-1$. Meanwhile, the damping $\mathbf{K}_{d}$ is kept proportional to the proportional gain

$$
\mathbf{K}_{d}^{k}=\alpha \mathbf{K}_{p}^{k} .
$$

Both gains are saturated between $\mathbf{K}_{\max }$ and $\mathbf{K}_{\min }>0$, with

$$
\alpha=\mathbf{K}_{d}^{\max } / \mathbf{K}_{p}^{\max } .
$$

\section{EXPERIMENT}

\section{A. Robot}

The adaptive algorithm was coded on the real time controller (RTLinux running control loop at $1 \mathrm{kHz}$ ) of an ABLE exoskeleton from CEA-LIST [12], a four active degrees of freedom (DOF) robot, with 3-DOF at the shoulder (for abduction/adduction, internal/external rotation, and flexion/extension) and one at the elbow (for flexion/extension). ABLE is based on a patented screw-cable mechanical transmission [13] which, together with the model-based gravity compensation, allows high backdriveability of the device.

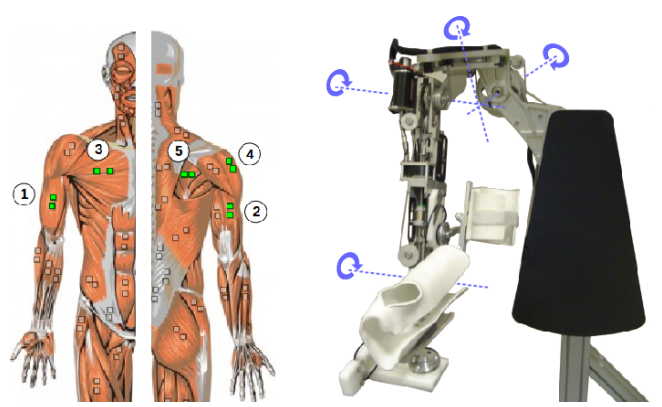

Fig. 1. Left: EMG sensor positions (from Noraxon@MyoResearchXP). Muscles: (1) biceps, (2) lateral triceps, (3) pectoralis major, (4) middle deltoid, and (5) infraspinatus. Right: ABLE 4-DOF exoskeleton.

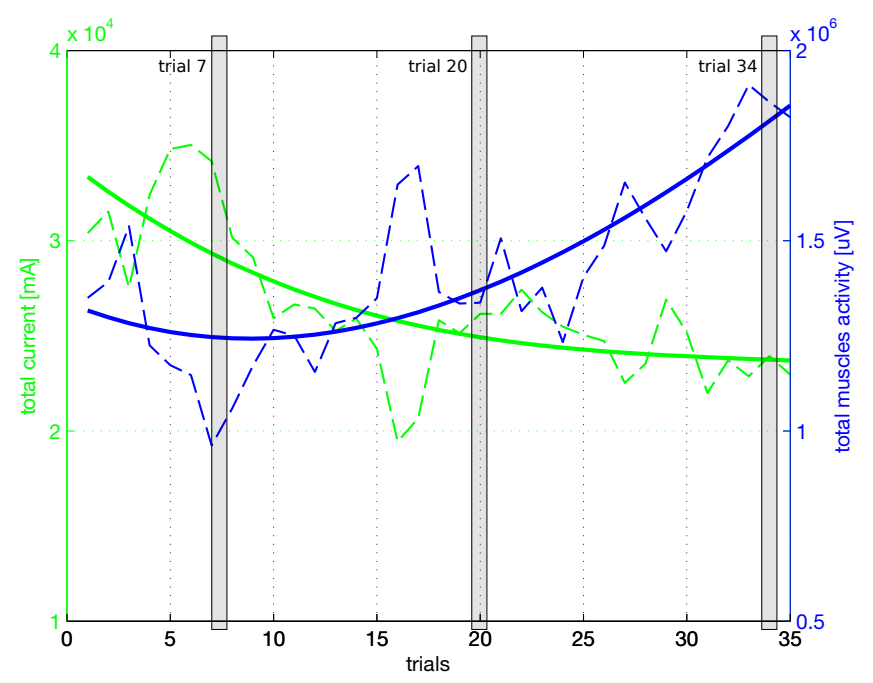

Fig. 2. Muscles activity (dashed blue line) and total current from the adaptive PD controller (dashed green line) over the 35 trials. The two solid lines are the two terms tendencies computed through linear regression. See figure 4 for details of trial 7, 20, and 34 .

\section{B. Protocol}

We tested our controller on one healthy subject. The experiment consisted in mimicking a simplified process of relearning by a post-stroke patient who undergoes neurorehabilitation therapy. The main difference with proper neurorehabilitation was the duration of the therapy, since the learning occurred in just 35 trials. In this number of trials, the healthy subject was asked to perform the desired motion task with different level of muscle activity, in particular starting from zero activity, i.e. being fully relaxed, mimicking the incapacity to perform the motion at all, to full control of his limb, mimicking the ideal end of the relearning therapy. We recorded the activity of five groups of muscles through EMG, in particular biceps, lateral triceps, pectoralis major, middle deltoid, and infraspinatus, see figure 1 . This measure was not used in the control loop, but it was visually provided in real-time to the subject to have a direct feedback of his level of activity. The EMG was recorded at $1.5 \mathrm{KHz}$ with Noraxon๑TeleMyo DTS system and the resulting values were passband filtered between $10 \mathrm{~Hz}$ and $400 \mathrm{~Hz}$, offsetted (its mean value was removed), rectified, and finally processed 


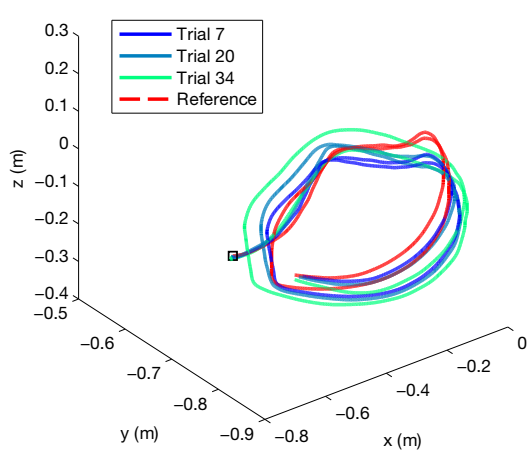

Fig. 3. End-effector trajectories. The small square shows the starting point, in red the reference trajectory. Blue line is trial 7, dark green trial 20, and light green trial 34 .

with hilbert envelop function on Matlab@ $\odot$. The motion task was simply to draw in the air in front of the subject two 4 edges polygons of side about $50 \mathrm{~cm}$ as shown in figure 3 by the red line. The trajectory lasted approximatively $10 \mathrm{~s}$. No markers or any other visual feedback related to the desired trajectory were provided to the subject in order to make the learning process more difficult to be successfully achieved. All the recorded data from ABLE exoskeleton were passband filtered during post-processing using 3rd order butterworth filter. For our experiment we chose the following values $\beta=0.25, \gamma=5, \mathbf{K}_{p}^{\max }=[300,200,200,200]$, $\mathbf{K}_{p}^{\text {min }}=[10,10,10,10], \mathbf{K}_{d}^{\max }=[10,10,10,10]$, and $\mathbf{K}_{d}^{\min }=[1,1,1,1]$.

\section{Results}

After an initial training with the exoskeleton in transparency (null PD gains and active gravity compensation), we recorded 35 trials with the adaptive controller on, asking the subject to perform the experiment motion task with increasing level of muscles activation. In particular the subject was asked to perform the first third of trials with $0 \%$ of activation, i.e. staying relaxed and leaving the exoskeleton working for him, then the second third of trials with $50 \%$ of activation, i.e. sharing the effort of the motion with the controller, and finally to perform the task at his best, as a patient who has finally relearnt how to control his limb and does not need the exoskeleton anymore.

Figure 2 shows muscle activity $\Psi(t)=\sum_{i=1}^{5} \psi_{i}(t)$ and total current $\mathbf{V}=\sum_{i=1}^{4}\left(\mathbf{v}_{i} / \delta\right)$ over the 35 trials, where $\psi_{i}(t)$ is the single trial activity profile of one of the considered muscle groups, $\mathbf{v}_{i}$ the total current on the $i$-th actuator from the PD adaptive controller, and $\delta=r_{g} \tau \approx 4.26$ is the product of gear ratio $r_{g}$ and torque constant $\tau$. In the same figure, the two tendencies are obtained through 3rd order linear regression.

Three detailed trials, manually selected for the interesting resulting profiles, are shown in figure 4. First column is trial 7 , second trial 20, and third column is trial 34 . The first row of plots shows muscles activities through EMG measurement over the samples for the 5 different muscle groups. The second line of graphs describes PD controller torque outputs for the 4-DOF. Also, in the same plots, $K p$ and $K d$ values for the chosen trials. Finally, last row of graphs contains joint positions and desired positions for the 4-DOF.

\section{Discussion}

The aim of this controller is to provide an algorithm able to automatically adapt robot compliance by moving between passive and active-assistive mode depending on patient capacities. The adaptation of PD gains following the process of learning of the subject, is shown in figure 2 by the decrease of the necessary current. This figure shows how, as the muscles activity increases, meaning more subject involvement in achieving the task, the adaptive controller is becoming more transparent, meaning less constraining over the subject's limb. In particular, between trial 1 and trial 35 , total current decreases by $25 \%$ of its initial value. The trials details of figure 4 clearly show how the output of the adaptive PD controller decreased while the EMG data increased, corresponding to a less constrained and more involved subject. At the final trial, $\mathbf{K}_{p}=[132.8,40.248 .3,45.1]$ and $\mathbf{K}_{d}=$ $[4.4,2.0,2.4,2.2]$ gave to the subject enough transparency to perform the task almost freely. As expected, less control increased the position errors, which however remain small enough to be considered negligible even at the final trial (within 5 degree at the joint level, and an increase of approximatively $1 \mathrm{~cm}$ of the mean error at the end-effector between the first and the last trial).

The adaptive parameters $\beta$ and $\gamma$ can be tuned differently either to achieve a fast adaptation (high gains) meaning less assistance as soon as possible, or slow adaptation (low gains) meaning less controller modification between trials. More work is however necessary to define methods to tune these parameters in order to give a simple control of adaptation rate to physiotherapists.

\section{Conclusions}

We presented an adaptive technique for controlling robotic exoskeletons in neurorehabilitation. This algorithm exploits adaptation of control gains to allow for increasing subject involvement when he learns the task. The control law can automatically and continuously adapt between passive and active-assistive control mode, based on subject's performance. This is a new feature for neurorehabilitation because it is something which standard therapy cannot provide. Note that it requires to tune the rate of change of the controller based on subject disease phase, which is expected to be done by the physiotherapist based on his experience.

Initial results are encouraging but limited to only one healthy subject. Future research will mainly concern testing the paradigm on more healthy subjects, and if the results confirm efficient adaptation, to move on post-stroke patients.

Another interesting approach would be to develop simultaneous feedforward adaptation as in the motor control learning paradigm explained at the beginning of section II. This could lead to less impact of the feedback term on the overall control, leading to less oscillation, better disturbance suppression, and even less subject constraining. 
TRIAL 7
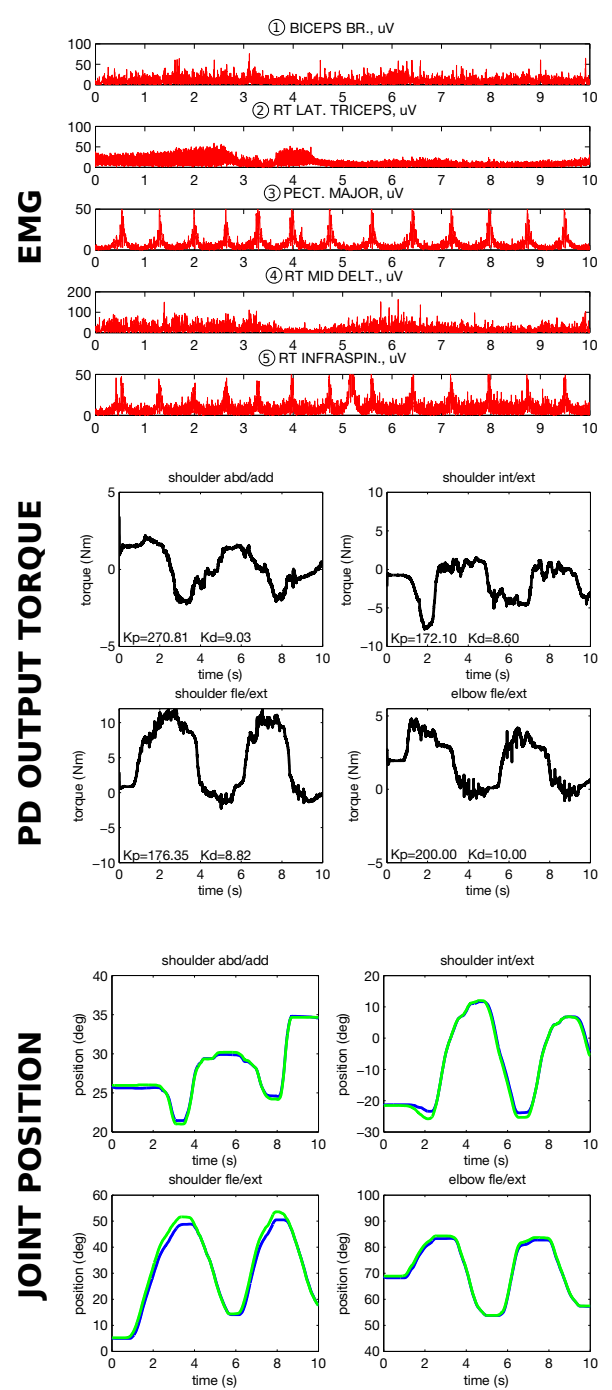

TRIAL 20
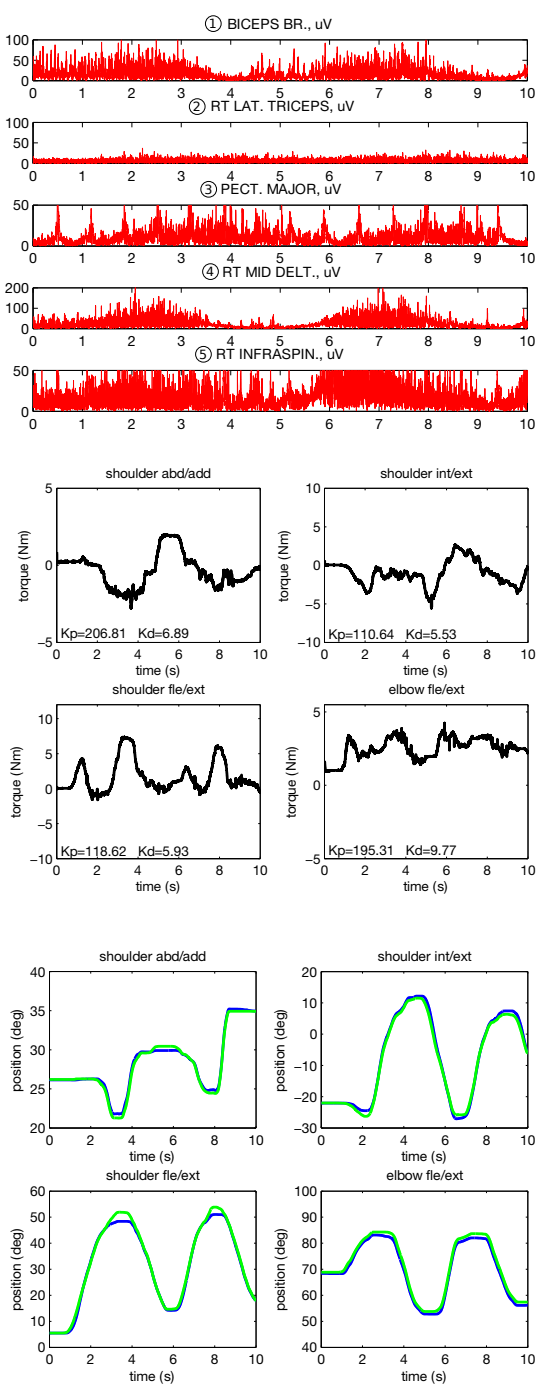

TRIAL 34
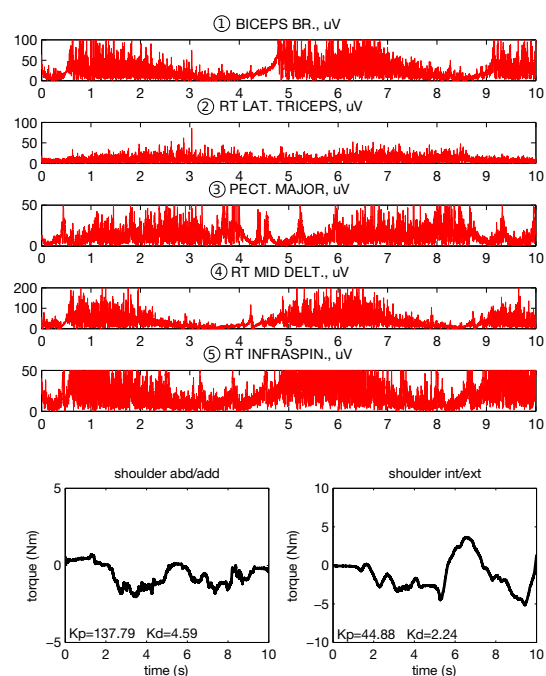

time (s)
shoulder fle/ext
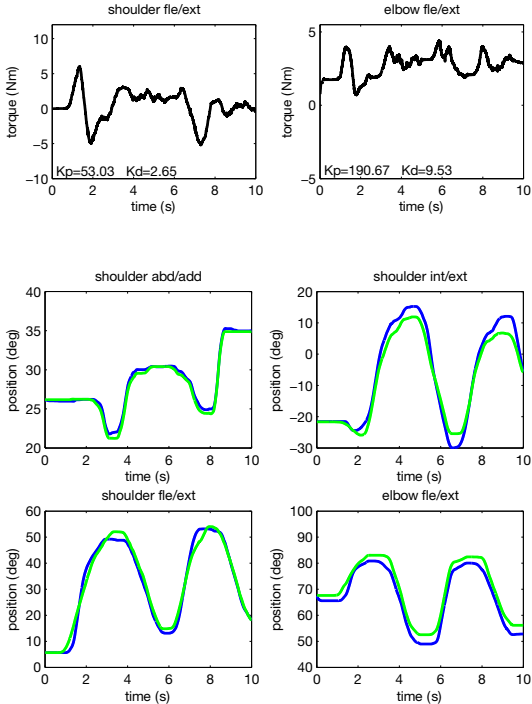

Fig. 4. Trials details. First column is trial 7 , second trial 20 , and third column is trial 34 . In red, muscles activity $[\mu V]$ during the trial, for the 5 different muscles (from top to bottom, (1) biceps, (2) lateral triceps, (3) pectoralis major, (4) middle deltoid, and (5) infraspinatus) over the samples (sampling frequency is $1.5 \mathrm{KHz}$ ). In black, PD controller torque output $[\mathrm{Nm}]$ for the 4-DOF (top-bottom, right-left, joint 1 shoulder abduction/adduction, joint 2 shoulder internal/external rotation, joint 3 shoulder flexion/extension, and joint 4 elbow flexion/extension). Also, in the same plots, $K p$ and $K d$ values for the chosen trials are shown. Finally, last line of graphs presents in blue joint position $[\mathrm{deg}]$ and in green joint desired position [deg] for the 4-DOF. As expected, EMG activity and joint position error are increasing with the trials, while controller output is decreasing resulting in an increase of robot transparency.

\section{REFERENCES}

[1] Gresham Glen E., Pamela W. Duncan, and William B. Stason, Poststroke rehabilitation, Vol. 95, No. 662, DIANE Publishing, 1997

[2] Burdet, Etienne, David W. Franklin, and Theodore E. Milner, Human Robotics: Neuromechanics and Motor Control, MIT Press, 2013

[3] Lynch D., Ferraro M., Krol J., Trudell C. M., Christos P., and Volpe B. T., Continuous passive motion improves shoulder joint integrity following stroke, Clinical Rehabilitation, 2005, 19, 6, 594599

[4] Mao Ying, and Sunil Kumar Agrawal, A cable driven upper arm exoskeleton for upper extremity rehabilitation, Robotics and Automation (ICRA), 2011 IEEE International Conference on. IEEE, 2011

[5] Krebs. et al., Motions or muscles? Some behavioral factors underlying robotic assistance of motor recovery, J Rehabil. Res. Dev., 2006, 43, 5,605618

[6] Patton J. L. and Mussa-Ivaldi F. A., Robot assisted adaptative training: Custom force fields for teaching movement patterns, IEEE Rev. Biomed. Eng., 2002, 51, 636646

[7] Hogan N. and Krebs H. I., Interactive robots for neuro-rehabilitation, Restorative Neurology and Neuroscience, 2004, 22, 3-5, 349358
[8] Wolbrecht E.T., Chan V., Reinkensmeyer D.J., and Bobrow J.E., Optimizing Compliant, Model-Based Robotic Assistance to Promote Neurorehabilitation, IEEE Transactions on Neural Systems and Rehabilitation Engineering 16, no. 3 (June 2008): 28697

[9] Wei et al., Adaptive Iterative Learning Control Design for RUPERT IV, 64752, 2008

[10] Gopura et al., SUEFUL-7: A 7DOF upper-limb exoskeleton robot with muscle-model-oriented EMG-based control, Intelligent Robots and Systems, 2009. IROS 2009. IEEE/RSJ International Conference on. IEEE, 2009

[11] Franklin D. W., E. Burdet, K. Peng Tee, R. Osu, C.-M. Chew, T. E. Milner, and M. Kawato, CNS Learns Stable, Accurate, and Efficient Movements Using a Simple Algorithm, Journal of Neuroscience 28, no. 44 (October 29, 2008): 1116573

[12] Garrec P. et al., ABLE, an innovative transparent exoskeleton for the upper-limb, IEEE/RSJ International Conference on Intelligent Robots and Systems IROS, 2008, pp. 1483-1488

[13] Garrec P., CEA - European patent EUR 01938347.02421-FR0101630: Nut-screw and cable transmission 